\title{
Association of Monocyte-Lymphocyte Ratio and Proliferative Diabetic Retinopathy in the U.S. Population with Diabetic Retinopathy
}

\section{Wang Huan ( $\sim 13652026874 @ 163 . c o m$ )}

Tianjin Children's Hospital

\section{Guo Zhen}

Tianjin Children's Hospital

\section{Xu Yu}

Tianjin Children's Hospital

\section{Research Article}

Keywords: Diabetic retinopathy, proliferative diabetic retinopathy, monocyte count/lymphocyte count, diabetic patients

Posted Date: November 18th, 2021

DOI: https://doi.org/10.21203/rs.3.rs-992972/v1

License: (a) (i) This work is licensed under a Creative Commons Attribution 4.0 International License. Read Full License 


\section{Abstract}

Diabetic retinopathy (DR) is one of the most common causes of blindness and visual impairment, especially proliferative diabetic retinopathy (PDR). Early prediction of its occurrence and progression is important. However, the association between monocyte-lymphocyte ratio(MLR) and PDR remains unclear. This study aimed to investigate the association between MLR and PDR in the U.S. population with DR from NHANES 2005 to 2008. DR was defined by the criteria of the Early Treatment for Diabetic Retinopathy Study based on nonmydriatic fundus photography. The MLR is the monocyte count/lymphocyte count. The lymphocyte count and monocyte count can be obtained directly from laboratory data files. Logistic regression was applied to explore the association between MLR and PDR. A total of 300 participants were included, the prevalence of PDR was $9 \%(27 / 300)$. In the multivariate regression models, patients with PDR was significantly associated with MLR (adjusted OR $=1.9,95 \% \mathrm{Cl}: 1.26,2.84$ ) after adjusting all of the covariates. The interaction analysis showed an interactive role in the association between MLR and PDR by PIR(P for interaction=0.02). MLR were significantly associated with the occurrence of PDR in diabetic patients. The assessment of MLR levels might be a part of follow-up visits with diabetic patients.

\section{Introduction}

Diabetic retinopathy (DR) is suggested to be a leading cause of blindness, and it contributes to $2.6 \%$ and $1.9 \%$ of visual impairmen and tblindness in worldwide ${ }^{[1]}$. The prevalence of DR is approximately $33 \%$ in western countries ${ }^{[2]}$. Therefore, early identification of the microvascular complication risks provide an opportunity to delay or stop disease onset ${ }^{[3]}$. Some studies have shown that many biomarkers can reflect the presence of microvascular complications ${ }^{[4]}$, and they are also associated with the risk of retinopathy ${ }^{[5]}$.

Increasing evidence points that many inflammatory markers are associated with increased risk of $\mathrm{DR}^{[6]}$, and inflammation-related markers would play important roles in prediction and disease assessment of DR. Monocyte-lymphocytes ratio (MLR) is a novel inflammatory marker, and plays an important role in prediction and prognosis of some inflammation-related diseases, such as tumors, cardiovascular diseases and DR ${ }^{[7]}$. Some previous studies have showed that WBC sub-types were closely associated with inflammatory state of $\mathrm{DR}^{[8]}$. We speculated that MLR may play important roles in development and progression of $\mathrm{DR}$, and it would had a valuable significance in PDR patients. However, the associations of MLR with PDR is unknown. Thus, the aim of this study was to explore the clinical and predictive significance of MLR in PDR patients.

\section{Methods}

\section{Study Population}

The National Health and Nutrition Examination Survey (NHANES) is executed by the National Center for Health Statistics (NCHS), which contains these US noninstitutionalized civilian participants. All participants underwent comprehensive measurements, such as physical and laboratory examinations and standardized interview questionnaires, including socioeconomic, demographic, and health-related questions. 
In our study, we used public data from two NHANES cycles (2005-2006, 2007-2008). More informations about the data are available on the NHANES website (www.cdc.gov/nchs/nhanes/). Between 2005 and 2008 , there were 20,497 participants in NHANES. We first excluded 19,199 participants with no diabetes. The subsequent exclusion criteria were as follows: (I) unknow retinopathy grading ( $n=414)$; (II) unknown peripheral blood MLR ( $n=46)$; (III) no diabetic retinopathy $(n=538)$. After excluding these factors, 300 participants were included in the final study.

According to the Declaration of Helsinki, this design was approved by the institutional review board of the NCHS. Before examinations, all participants completed informed consents.

\section{Study variables and outcome}

The MLR is the monocyte count/lymphocyte count. The lymphocyte count and monocyte count can be obtained directly from laboratory data files. The neutrophil count is calculated from the white blood cell count and neutrophil percentage.

$\mathrm{DR}^{[9]}$ was defined by the presence of hemorrhages, hard exudates (HE), cotton wool spots (CWS), microaneurysms (MA), venous beading, intraretinal microvascular abnormalities (IRMA), and retinal new vessels based on the severity scale of the Early Treatment for Diabetic Retinopathy Study (ETDRS). Nonmydriatic fundus photography (TRC-NW6S; Topcon, Tokyo, Japan) was applied for measuring the level of retinopathy in the worse eye. The grades were categorized into no DR, non-proliferative DR, and proliferative DR. Detailed information is listed in the Digital Grading Protocol of the NHANES.

Other covariates included sex (male or female), age, race (non-Hispanic white, non-Hispanic Black, Mexican American, other Hispanic and other), marital status (married, unmarried and other), poverty income ratio ( $<1$, $\geq 1$ ), education level (less than high school, high school or equivalent, college or above and other), BMI (<25.0, 25.0-29.9 and $\geq 30.0 \mathrm{~kg} / \mathrm{m} 2)$, cotinine (<0.015, 0.015-10, and $\geq 10), \mathrm{HbA} 1 \mathrm{C}(<7 \%, \geq 7 \%)$. Diabetes are defined as self-reported physician-diagnosed diabetes ${ }^{[10]}$. Duration of diabetes ${ }^{[11]}$ was calculated from the reported age at screening minus the age of the subject when first told he/she had diabetes. Family history of diabetes was determined by the answer to the following question: 'Including living and deceased, were any of your biological relatives, that is, blood relatives, including grandparents, parents, brothers, and sisters, ever told by a health professional that they had diabetes?'

\section{Statistical Analysis}

All the analyses were performed with the statistical software packages R (http://www.R-project.org, The R Foundation) and Free Statistics software versions 1.3. The differences of continuous and categorical variables were investigated using the independent-test and the chi-squared test, respectively. These logistic regression models were used to determine the relationship of MLR with the presence of PDR. Model 1 was unadjusted. Model 2 was adjusted by age, sex, race, PIR. Model 3 was Model 2 + adjusted by BMI, Cotinine, total cholesterol, HGB, Model 4 was Model 3 + adjusted by HbA1C, duration of diabetes. Subgroup analysis examined the relationship between MLR and PDR according to PIR. Test for interaction in the logistic regression model was used to compare odd ratios (ORs) between the analyzed subgroups. 


\section{Results}

\section{Study population characteristics}

Based on the inclusion and exclusion criteria in Fig. 1, we selected 300 qualified participants from NHANES 2005-2008. PDR was detected in 27 subjects (9\%). Table 1 shows the demographic, socioeconomic, comorbidity and baseline characteristics by PDR and NPR. Significant differences in PIR, cotinine, HGB and duration of diabetes were observed between PDR and NPR (all P-valueso0.05). The lower levels of PIR and HGB were present in the group of subjects with PDR $(P=0.012,0.002)$. Subjects with PDR had the longest duration of diabetes (21.6 years, $P=0.002$ ) and with NPR (14.8 years). A higher percentage of the lowest levels of cotinine in the PDR group (33.3\%) compared with $17 \%$ for NPR $(P=0.043)$.

\section{Factors associated with PDR}

Regression analysis was performed to identify factors in the entire study population that were associated with PDR. The result of univariate ordinal regression analysis indicates PIR, ethnicity (other), cotinine (high), $\mathrm{HGB}$, and duration of diabetes were positively associated with PDR (all $\mathrm{P}<0.05$, Table 2 ).

\section{Association between MLR and the Presence of PDR}

In the multivariate regression models, MLR was positively associated with the presence of PDR with an odds ratio (OR) of 1.38 (95\% confidence interval (Cl): 1.1-1.75), 1.74 (95\% Cl: 1.28-2.38), 1.6 (95\% Cl: 1.14-2.24), 1.9 (95\% Cl: 1.26-2.84) in Model 1, Model 2, Model 3 and Model 4, respectively (Table 3).

In addition, after adjusting all of the covariates, the interaction of MLR with PIR significantly affected the Presence of PDR $(P=0.002)$

The interaction analysis revealed that PIR played an interactive role in the association between MLR and the presence of PDR. PIR< 1 subgroup had higher OR between MLR and the presence of PDR $(\mathrm{OR}=5.7 ; 95 \% \mathrm{Cl}$; 1.05 - 30.8; $P=0.043)$ than $P I R \geq 1$ subgroup $(\mathrm{OR}=1.74 ; 95 \% \mathrm{Cl}, 1.05-2.89 ; \mathrm{p}=0.032)$ (Table4, Fig 2).

\section{Discussion}

In this study, we used the NHANES database. Our results showed for the first time that the MLR increased as the incidence of PDR increased. There was a significant correlation between MLR and PDR. After adjusting for other confounding factors, MLR was associated with the prevalence of PDR. This indicates that the MLR might be used as a predictor for the occurrence and progression of PDR.

Increasing evidences have showed that there are a dominant role of chronic inflammation in the development of diabetic retinopathy ${ }^{[12]}$. Grossmann et al. pointed that WBCs, granulocytes, and monocytes except the lymphocyte gradually increased from normoglycemic subjects to subjects with diabetes ${ }^{[13]}$. And Ji et al. reported that monocyte to lymphocyte ratio (MLR) or lymphocyte to monocyte ratio (LMR) could mirror the circulating immune status of the host ${ }^{[14]}$. The MLR level may have better stability than independent monocyte, lymphocyte and leukocyte levels because of the balance between the monocyte and lymphocyte 
levels which is less affected by various physiologica and Ipathological status. The MLR has been considered a novel inflammatory biomarker as a readily available and inexpensive index calculated by blood routine examination. Therefore, MLR might be a good reflection of different clinical conditions in DR patients.

Qinghua Huang et al. showed that a significantly higher MLR in DR patient with proliferative stage than that with non-proliferative stage, and the MLR is a powerful predictor for the occurrence of $D^{[15]}$. Song Yue et al. suggested that higher MLR values may be an independent risk factor for $\mathrm{DR}^{[16]}$. Some studies shouwed that the increasing of the MLR may be associated with the production of the pro-inflammatory chemokines such as IL-6, TNF- $a$, IL-1 $\beta$, and MCP- 1 . They play major roles in the recruitment and activation of monocytes and leukocytes, and the subsequent inflammatory responses in DR patients ${ }^{[6,15]}$. However, the relationship between PDR and MLR has not been investigated so far. In our study, we found that PDR patients had remarkably higher MLR than NPDR patients. Because of an enhanced inflammatory response and a reduced immune function, a high MLR in PDR patient may be resulted from an increased number of monocytes and a decreased number of lymphocytes. Therefore, the findings suggest that MLR is closely associated with the risk of PDR.

Heng Wan et al. Showed that the low peripheral blood monocyte levels as a biomarker can screen the early stage of $D R$, but the level of neutrophils and lymphocytes cannot be a biomarker ${ }^{[17]}$. There are two ways to attracting and influxing monocytes into the retina. One is adhering to the outer surface of retinal capillaries, and the other is breaking down the blood-retinal barrier. Finally, it may decrease the monocyte level in the peripheral blood. The controversial results may be resulted from the different conditions of the participants, such as subject heterogeneity and lifestyle difference.

We found that MLR was significantly higher in $D R$ patients with $P I R<1$ group than $P I R \geq 1$ group. A higher family poverty income ratio was associated with the presence of PDR. It is likely that many low-income participants were not able to afford the recommended treatment of diabetes. Inorder to confirm these findings, additional studies are needed.

There are some limitations to this study. First, our study is based on the NHANES database, which is a crosssectional study; further research requires a prospective study. Second, there is no information in the NHANES database about the type of diabetes. Moreover, NHANES survey results are based on participants' selfreported data and require the use of a diagnostic test. Third, even though a relationship between the MLR and the presence of PDR was established, the causal relation could not be addressed due to the study's crosssectional design.

\section{Conclusions}

The study investigated the relationship between MLR and the occurrence of PDR. The MLR is significantly increased in PDR patients after adjusting for confounding variables. The MLR is a convenient and economical biomarker derived from a routine blood examination, it may play an important role in follow-up visits in diabetic patients. Additional studies are needed to identify the mechanism explaining the association between the MLR and PDR. 


\section{Declarations}

The findings and conclusions in this report are those of the authors and do not necessarily

represent the official position of the Centers for Disease Control and Prevention.

Data availability The data analyzed in the current study are publicly available.

Funding/Support : No funding.

Financial Disclosures : No financial disclosures.

\section{Acknowledgments}

We thank Dr.LiuJie (People's Liberation Army of China Genernal Hospital, Beijing, China) and Dr. Yang Qilin (The Second Affiliated Hospital of Guangzhou Medical University, Guangzhou, Guangdong, China) for helping in this revision

\section{References}

1. BOURNE R R, STEVENS G A, WHITE R A, et al. Causes of vision loss worldwide, 1990-2010: a systematic analysis[J]. Lancet Glob Health, 2013, 1(6): e339-349.

2. WONG T Y, KLEIN R, ISLAM F M, et al. Diabetic retinopathy in a multi-ethnic cohort in the United States[J]. Am J Ophthalmol, 2006, 141(3): 446-455.

3. ABBASI A, PEELEN L M, CORPELEIJN E, et al. Prediction models for risk of developing type 2 diabetes: systematic literature search and independent external validation study[J]. Bmj, 2012, 345: e5900.

4. HO H, CHEUNG C Y, SABANAYAGAM C, et al. Retinopathy Signs Improved Prediction and Reclassification of Cardiovascular Disease Risk in Diabetes: A prospective cohort study[J]. Sci Rep, 2017, 7: 41492.

5. TOTH P P, SIMKO R J, PALLI S R, et al. The impact of serum lipids on risk for microangiopathy in patients with type 2 diabetes mellitus[J]. Cardiovasc Diabetol, 2012, 11: 109.

6. RüBSAM A, PARIKH S, FORT P E. Role of Inflammation in Diabetic Retinopathy[J]. Int J Mol Sci, 2018, 19(4).

7. LIU J, LIU X, LI Y, et al. The association of neutrophil to lymphocyte ratio, mean platelet volume, and platelet distribution width with diabetic retinopathy and nephropathy: a meta-analysis[J]. Biosci Rep, 2018, 38(3).

8. YUAN C, LI N, MAO X, et al. Elevated pretreatment neutrophil/white blood cell ratio and monocyte/lymphocyte ratio predict poor survival in patients with curatively resected non-small cell lung cancer: Results from a large cohort[J]. Thorac Cancer, 2017, 8(4): 350-358.

9. CHEN Y J, CHEN J T, TAI M C, et al. Serum Iron and Risk of Diabetic Retinopathy[J]. Nutrients, 2020, 12(8).

10. MAO W, WU J, ZHANG Z, et al. Neutrophil-lymphocyte ratio acts as a novel diagnostic biomarker for kidney stone prevalence and number of stones passed[J]. Transl Androl Urol, 2021, 10(1): 77-86. 
11. LONG M, WANG C, LIU D. Glycated hemoglobin A1C and vitamin D and their association with diabetic retinopathy severity[J]. Nutr Diabetes, 2017, 7(6): e281.

12. FUJITA T, HEMMI S, KAJIWARA M, et al. Complement-mediated chronic inflammation is associated with diabetic microvascular complication[J]. Diabetes Metab Res Rev, 2013, 29(3): 220-226.

13. GROSSMANN V, SCHMITT V H, ZELLER T, et al. Profile of the Immune and Inflammatory Response in Individuals With Prediabetes and Type 2 Diabetes[J]. Diabetes Care, 2015, 38(7): 1356-1364.

14. JI H, NIU X, YIN L, et al. Ratio of Immune Response to Tumor Burden Predicts Survival Via Regulating Functions of Lymphocytes and Monocytes in Diffuse Large B-Cell Lymphoma[J]. Cell Physiol Biochem, 2018, 45(3): 951-961.

15. HUANG Q, WU H, WO M, et al. Clinical and predictive significance of Plasma Fibrinogen Concentrations combined Monocyte-lymphocyte ratio in patients with Diabetic Retinopathy[J]. Int J Med Sci, 2021, 18(6): 1390-1398.

16. YUE S, ZHANG J, WU J, et al. Use of the Monocyte-to-Lymphocyte Ratio to Predict Diabetic Retinopathy[J]. Int J Environ Res Public Health, 2015, 12(8): 10009-10019.

17. WAN H, CAI Y, WANG Y, et al. The unique association between the level of peripheral blood monocytes and the prevalence of diabetic retinopathy: a cross-sectional study[J]. J Transl Med, 2020, 18(1): 248.

18. BENHAR I, REEMST K, KALCHENKO V, et al. The retinal pigment epithelium as a gateway for monocyte trafficking into the eye[J]. Embo j, 2016, 35(11): 1219-1235.

\section{Tables}

Table 1. Baseline characteristics of patients 


\begin{tabular}{|c|c|c|c|c|}
\hline Characteristics & Total & NPDR & PDR & \\
\hline $\mathrm{n}$ & 300 & 273 & 27 & $\mathrm{p}$ \\
\hline age (years) & $63.9 \pm 10.5$ & $63.9 \pm 10.7$ & $63.0 \pm 7.8$ & 0.655 \\
\hline sex, n (\%) & & & & 0.174 \\
\hline male & $165(55.0)$ & $154(56.4)$ & $11(40.7)$ & \\
\hline female & $135(45.0)$ & $119(43.6)$ & $16(59.3)$ & \\
\hline Race/ethnicity, n (\%) & & & & 0.100 \\
\hline Non-Hispanic white & $108(36.0)$ & $103(37.7)$ & $5(18.5)$ & \\
\hline Non-Hispanic black & $105(35.0)$ & $93(34.1)$ & $12(44.4)$ & \\
\hline Mexican American & $58(19.3)$ & $53(19.4)$ & $5(18.5)$ & \\
\hline Other & $29(9.7)$ & $24(8.8)$ & $5(18.5)$ & \\
\hline marriage, n (\%) & & & & 0.189 \\
\hline married & $185(61.7)$ & $172(63)$ & $13(48.1)$ & \\
\hline unmarried & $12(4.0)$ & $10(3.7)$ & $2(7.4)$ & \\
\hline other & $103(34.3)$ & $91(33.3)$ & $12(44.4)$ & \\
\hline PIR & $2.4 \pm 1.5$ & $2.5 \pm 1.5$ & $1.7 \pm 1.2$ & 0.012 \\
\hline BMI, n (\%) & & & & 0.171 \\
\hline Underweight/normal & $40(13.4)$ & 35 (12.8) & $5(19.2)$ & \\
\hline Overweight & $102(34.1)$ & $97(35.5)$ & $5(19.2)$ & \\
\hline Obese & $157(52.5)$ & $141(51.6)$ & $16(61.5)$ & \\
\hline Cotinine, n (\%) & & & & 0.043 \\
\hline$<\operatorname{LOD}(<0.015)$ & $55(18.5)$ & $46(17)$ & $9(33.3)$ & \\
\hline Low $(\geq 0.015-10)$ & $179(60.1)$ & $163(60.1)$ & $16(59.3)$ & \\
\hline $\operatorname{High}(\geq 10)$ & $64(21.5)$ & $62(22.9)$ & $2(7.4)$ & \\
\hline HbA1C poor control, n (\%) & & & & 0.370 \\
\hline no & $98(32.9)$ & $92(33.8)$ & $6(23.1)$ & \\
\hline yes & $200(67.1)$ & $180(66.2)$ & $20(76.9)$ & \\
\hline $\mathrm{HGB}(\mathrm{g} / \mathrm{dL})$ & $13.6 \pm 1.7$ & $13.7 \pm 1.7$ & $12.7 \pm 1.4$ & 0.002 \\
\hline CRP & $0.2(0.1,0.7)$ & $0.2(0.1,0.6)$ & $0.3(0.2,1.3)$ & 0.188 \\
\hline $\mathrm{HDL}(\mathrm{mmol} / \mathrm{L})$ & $1.3 \pm 0.4$ & $1.3 \pm 0.4$ & $1.3 \pm 0.4$ & 0.567 \\
\hline
\end{tabular}




\begin{tabular}{lllll} 
Total cholesterol(mmol/L) & $4.8 \pm 1.2$ & $4.8 \pm 1.2$ & $5.2 \pm 1.2$ & 0.069 \\
\hline Family history of diabetes, $\mathrm{n}(\%)$ & $206(68.7)$ & $185(67.8)$ & $21(77.8)$ & 0.582 \\
\hline Duration of diabetes (years) & $15.4 \pm 11.0$ & $14.8 \pm 11.1$ & $21.6 \pm 8.8$ & 0.002
\end{tabular}

Abbreviations: PIR, a ratio of family income to poverty threshold; BMI, body mass index; HGB, hemoglobin; $\mathrm{HDL}$, high-density lipoprotein cholesterol.

Table 2. Univariate analysis for the presence of PDR 
Characteristics

\begin{tabular}{|c|c|c|}
\hline $\mathrm{n}$ & OR $(95 \% \mathrm{Cl})$ & $P$ \\
\hline age (years) & $0.99(0.95 \sim 1.03)$ & 0.654 \\
\hline sex, n (\%) & & 0.123 \\
\hline male & 1 & \\
\hline female & $1.88(0.84 \sim 4.21)$ & \\
\hline \multicolumn{3}{|l|}{ Race/ethnicity, n (\%) } \\
\hline Non-Hispanic white & 1 & \\
\hline Non-Hispanic black & $2.66(0.9 \sim 7.83)$ & 0.076 \\
\hline Mexican American & $1.94(0.54 \sim 7.01)$ & 0.310 \\
\hline Other & $4.29(1.15 \sim 16.01)$ & 0.030 \\
\hline \multicolumn{3}{|l|}{ marriage, n (\%) } \\
\hline married & 1 & \\
\hline unmarried & $2.65(0.52 \sim 13.36)$ & 0.239 \\
\hline other & $1.74(0.76 \sim 3.98)$ & 0.186 \\
\hline PIR & $0.64(0.44 \sim 0.92)$ & 0.017 \\
\hline \multicolumn{3}{|l|}{ BMI } \\
\hline Underweight/normal & 1 & \\
\hline Overweight & $0.36(0.1 \sim 1.32)$ & 0.124 \\
\hline Obese & $0.79(0.27 \sim 2.32)$ & 0.673 \\
\hline \multicolumn{3}{|l|}{ Cotinine(ng/ml) } \\
\hline$<\operatorname{LOD}(<0.015)$ & 1 & \\
\hline Low $(\geq 0.015-10)$ & $0.5(0.21 \sim 1.21)$ & 0.124 \\
\hline High $(\geq 10)$ & $0.16(0.03 \sim 0.8)$ & 0.025 \\
\hline $\mathrm{HbA1C}$ & $1.13(0.92 \sim 1.4)$ & 0.248 \\
\hline $\mathrm{HGB}(\mathrm{g} / \mathrm{dL})$ & $0.7(0.55 \sim 0.88)$ & 0.003 \\
\hline CRP & $1.47(0.96 \sim 2.25)$ & 0.077 \\
\hline HDL (mmol/L) & $1.37(0.47 \sim 4.05)$ & 0.566 \\
\hline Total cholesterol(mmol/L) & $1.31(0.98 \sim 1.76)$ & 0.072 \\
\hline Family history of diabetes, n (\%) & $1.59(0.62 \sim 4.08)$ & 0.336 \\
\hline
\end{tabular}


Abbreviations: PIR, a ratio of family income to poverty threshold; BMI, body mass index; HGB, hemoglobin; HDL, high-density lipoprotein cholesterol.

Table 3. Association between MLR and the presence of PDR

\begin{tabular}{|c|c|c|c|c|c|c|c|c|}
\hline \multicolumn{9}{|c|}{$\operatorname{PDR}(\mathrm{n}=27)$} \\
\hline & model1 & & model2 & & model3 & & model 4 & \\
\hline & $\begin{array}{l}\mathrm{OR}(95 \% \\
\mathrm{Cl})\end{array}$ & $\mathrm{P}$ & OR $(95 \% \mathrm{Cl})$ & $\mathrm{P}$ & OR $(95 \% \mathrm{Cl})$ & $\mathrm{P}$ & OR $(95 \% \mathrm{Cl})$ & $\mathrm{P}$ \\
\hline$M L R * 10$ & $\begin{array}{l}1.38 \\
(1.1 \sim 1.75)\end{array}$ & 0.006 & $\begin{array}{l}1.74 \\
(1.28 \sim 2.38)\end{array}$ & $<0.001$ & $\begin{array}{l}1.6 \\
(1.14 \sim 2.24)\end{array}$ & 0.006 & $\begin{array}{l}1.9 \\
(1.26 \sim 2.84)\end{array}$ & 0.002 \\
\hline
\end{tabular}

Adjusted covariates: Model 1: unadjusted; Model 2: adjusted by age, sex, race, PIR; Model 3: Model $2+$ BMI, Cotinine, total cholesterol, HGB; Model 4: Model $3+\mathrm{HbA1C}$, duration of diabetes.

Table 4. Effect size of MLR on the presence of PDR in the PIR subgroup

\begin{tabular}{|c|c|c|c|c|c|c|c|}
\hline & \multirow[t]{2}{*}{$\mathrm{n}$} & \multirow[t]{2}{*}{$n(\%)$} & \multicolumn{2}{|l|}{ model1 } & \multicolumn{2}{|l|}{ model2 } & \multirow[t]{2}{*}{ P for interaction } \\
\hline & & & OR (95\% Cl) & $P$ & OR $(95 \% \mathrm{Cl})$ & $P$ & \\
\hline PIR & & & & & & & 0.020 \\
\hline$<1$ & 53 & $8(15.1)$ & $2.99(1.44 \sim 6.22)$ & 0.003 & $5.7(1.05 \sim 30.8)$ & 0.043 & \\
\hline$\geq 1$ & 219 & $16(7.3)$ & $1.31(0.99 \sim 1.74)$ & 0.060 & $1.74(1.05 \sim 2.89)$ & 0.032 & \\
\hline
\end{tabular}

Adjusted covariates: adjusted by age, sex, race, PIR, BMI, Cotinine, total cholesterol, HGB, HbA1C, duration of diabetes.

\section{Figures}




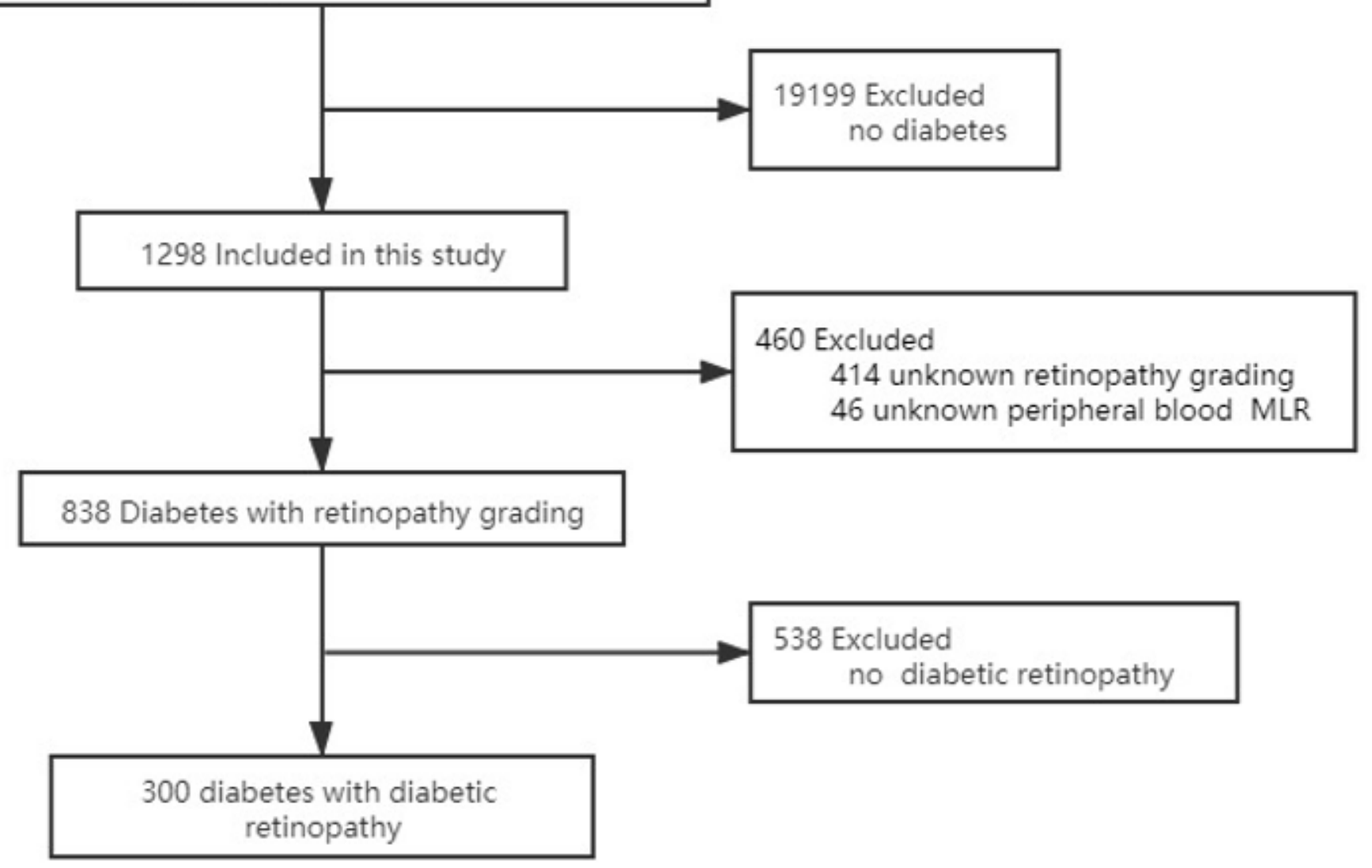

\section{Figure 1}

Schematic overview for patient identification 
n.total $n(\%)$

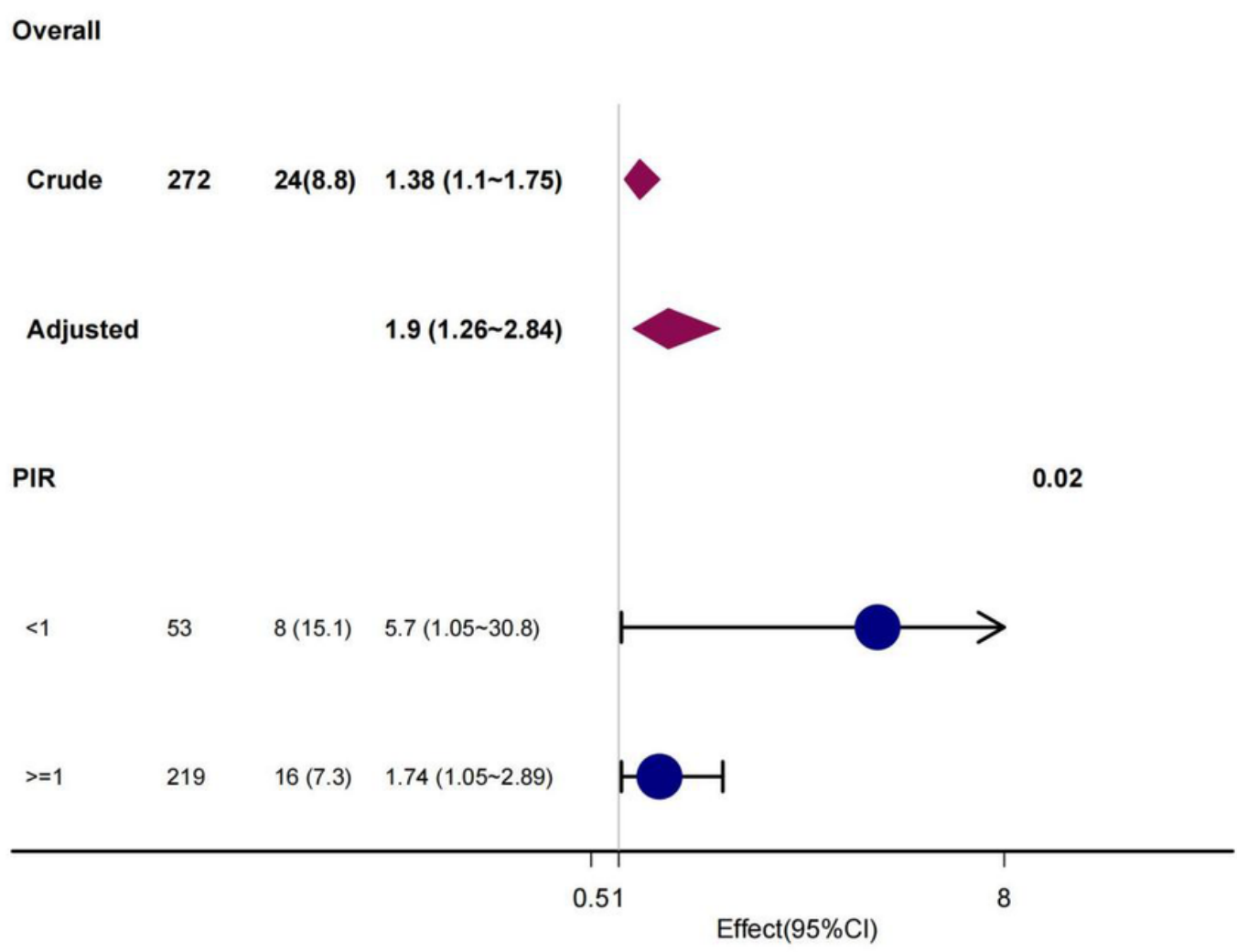

\section{Figure 2}

Effect size of MLR on the presence of PDR in the PIR subgroup 